\title{
Synthesis of silicon quantum dots using chitosan as a novel reductor agent
}

\author{
P. A. Hernández-Abril ${ }^{a, b}$, J. L. Iriqui-Razcón ${ }^{a}$, E. León-Sarabia ${ }^{a}$, S. D. Leal-Soto ${ }^{a}$, \\ M. E. Álvarez-Ramos ${ }^{b}$, D. Berman-Mendoza ${ }^{c}$ and H. J. Higuera-Valenzuela ${ }^{a, *}$ \\ ${ }^{a}$ Ingeniería Biomédica, Universidad Estatal de Sonora, \\ 83100 Hermosillo, Sonora, México. \\ ${ }^{b}$ Departamento de Física, Universidad de Sonora, \\ 83000 Hermosillo, Sonora, México. \\ ${ }^{c}$ Departamento de Investigación en Física, Universidad de Sonora, \\ 83000 Hermosillo, Sonora, México.
}

Received 28 July 2020; accepted 4 December 2020

\begin{abstract}
In the present paper we report a novel synthesis method of silicon quantum dots (SiQDs) using 3-Aminopropyltriethoxysilane (APTES) as silicon precursor and low molecular weight chitosan (CS) as reducing agent. The obtained SiQDs have a hydrodynamic diameter of 2.3 $\mathrm{nm}$, water dispersible, presents blue emission band at $434.5 \mathrm{~nm}(2.85 \mathrm{eV})$ with a Commission Internationale de l'Éclairage 1931 (CIE1931) chromaticity coordinates $(x=0.1665, y=0.1222)$, the experimental absorbance of the SiQDS was measured and the band gap (Eg) was estimated through PerkinElmer's method, for which the obtained value was $3.1 \mathrm{eV}$ and a positive $\zeta$-potential of $+35 \mathrm{mV}$, resulting in photonics, microelectronics, and biotechnological potential applications.
\end{abstract}

Keywords: Silicon quantum dots; chitosan; eco-friendly synthesis.

PACS: 81.07.Ta; 81.16.Be

DOI: https://doi.org/10.31349/RevMexFis.67.249

\section{Introduction}

Silicon ( $\mathrm{Si}$ ) has been successful as an active material in the electronic industry. Its characteristic indirect band gap has limited the applications based on the emission of light. However, this semiconductor has recently drawn the attention of researchers due to its novel properties in the nanometric scale, such as tunable photoluminescence response [1], low toxicity [2], and biocompatibility [3]. The photoluminescence (PL) studies of nanostructured silicon have increased since the emission at room temperature on porous silicon films [4]. Silicon quantum dots (SiQDs) have a wide range of potential applications; they have been used to improve the efficiency of solar cells [5], in the manufacture of light-emitting diodes (LED) [6], nonlinear optics, and secure communications cryptography [7]. The SiQDs have an extended fluorescence lifetime, according to multiples reports. This feature is particularly useful in cell imaging using fluorescencelife imaging microscopy [8] and bioimaging [9]. As a result, the amalgamation of these silicon quantum dots characteristics creates a new pathway for potential biomedical applications. Nowadays, silicon nanoparticles are commonly known as SiQDs. A significant breakthrough in this topic was the report that relates the luminescence from these SiQDs to their size and their electronic structure changes; the quantum confinement effect (QCE) is related to this phenomenon [10]. Therefore, investigations about new pathways for the synthesis of SiQDs have recently increased; the chemical and physical approaches are the core classification of synthesis techniques. The physical approach employs methods such as reactive sputtering [11], laser ablation [12], electrochemical etching and sonication [13], $\mathrm{SiO}_{2}$ implantation [14], and thermal annealing [15]. As a result, the PL emission for QDs is in the range between 500 and $950 \mathrm{~nm}$. On the other hand, the chemical approach applies processes as microemulsion synthesis [16], wet chemistry [17], synthesis in inverse micelles [18], and solution synthesis [19], which have produced QDs with an emission in the wavelength region from $300 \mathrm{~nm}$ to $500 \mathrm{~nm}[20]$.

In the search for new eco-friendly synthesis methods, one alternative is the replacement of the reducing agent. In previous investigations, polysaccharides as reducing agents in nanoparticles' synthesis have been reported [21-23]. Polysaccharides exhibit characteristics such as high biocompatibility [24,25], low cost [26-28] and easy processability $[29,30]$. Polysaccharides possess functional groups in their structure, including amines, carboxyl, and hydroxyl. These functional groups participate in REDOX reactions, the synthesis, and stabilization of metallic nanoparticles being one of the most common applications [31,32].

Biopolymers, such as chitosan, have been used to search for eco-friendly and biocompatible reducing agents to synthesize nanoparticles. The essential characteristics of CS are its high biocompatibility, water solubility, and the high response of its functional groups to $\mathrm{pH}$ [33]. The properties of chitosan are beneficial in applications such as water bioremediation [34], cell scaffolding [35], and controlled drug delivery [36]. The presence of functional groups such as $\mathrm{CH}_{2} \mathrm{OH}$ and $\mathrm{NH}_{2}$ gives it properties to reduce metallic salts to synthesize metallic nanoparticles [37,38]. 


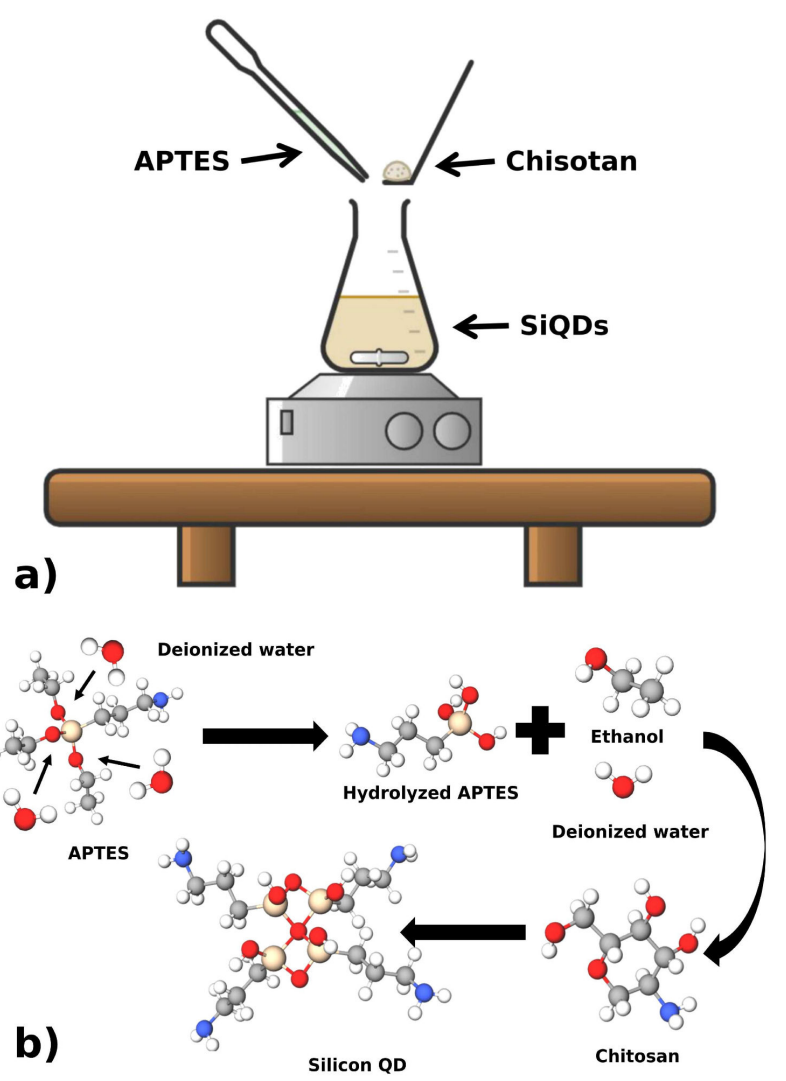

FIGURE 1. Schematic illustration a) and reaction b) of the synthesis of SiQDs.

\section{Experimental}

Figure 1a) shows the schematic illustration of the process.

The synthesis of SiQDs uses the method described by Wang, changing the reducing agent to CS. [39]. For the synthesis, the conditions were ambient temperature and atmospheric pressure.

The method consists of hydrolyzing APTES in deionized water under magnetic agitation for 10 minutes. Then, a solution of CS $(0.2 \mathrm{M})$ in deionized water was added to the previous mixture and stirred for an additional 20 minutes, as shown in Fig. 1b). In the next step, the resulting solution was centrifuged at 10000 RPM to separate the excess CS. The last step was collecting and storing supernatant for further characterization.

\section{Characterization}

The size distribution and $\zeta$-potential of the SiQDs were measured with a Malvern Zetasizer NanoSeries. The photoluminescence spectrum was obtained using a Kimmon IK Series He-Cd Laser of $325 \mathrm{~nm}$ and a HORIBA Scientific iHR320 Spectrometer. The UV-vis absorption spectrum was measured using a PerkinElmer UV/Vis/NIR Lambda 19 spectrometer.

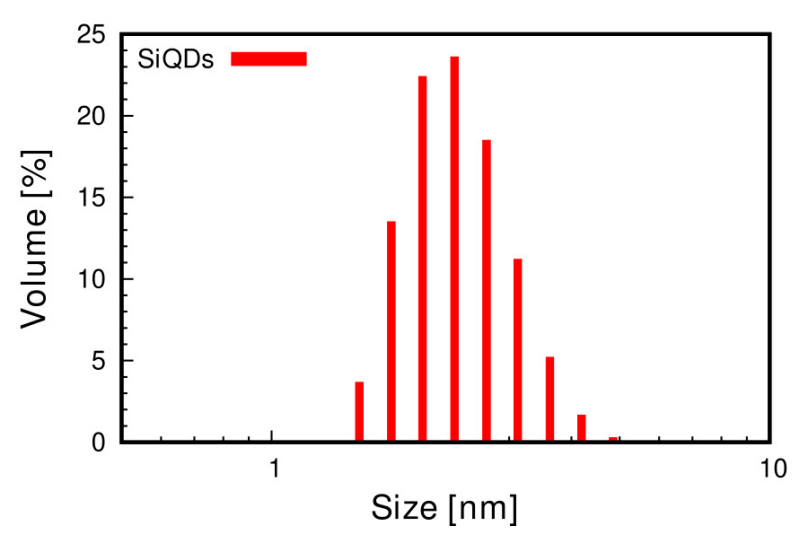

FIGURE 2. Size distribution of SiQDs.

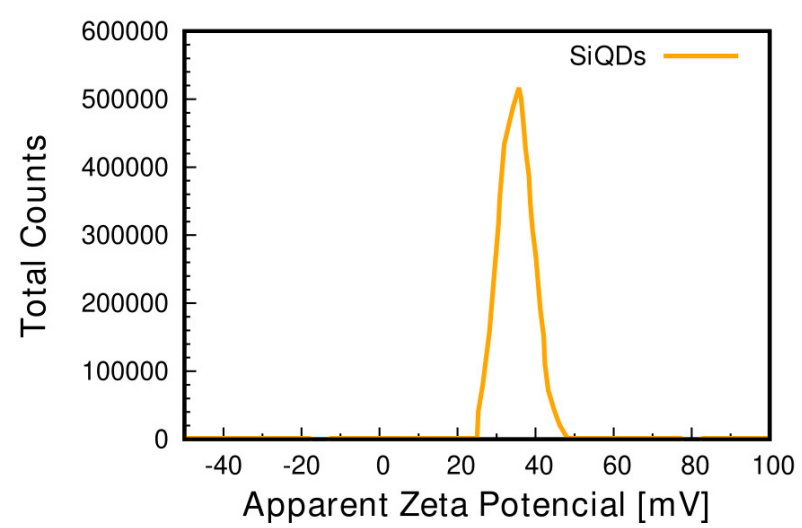

FIGURE 3. $\zeta$-potential of SiQDs.

\section{Results and discussion}

\subsection{Dynamic light scattering}

Figure 2 shown the hydrodynamic diameter of the quantum dots in a colloidal solution, with an average of $2.3 \mathrm{~nm}$. The size of the QDs is close to that shown in previous works to be highly biocompatible $[40,41]$. A small particle size allows the QDs to be internalized by pathways that do not include energy waste (pinocytosis [42] or endocytosis [43]), which is a desired characteristic in cell markers.

\section{2. $\zeta$-potential}

Figure 3 exhibits the SiQDs $\zeta$-potential of $+35 \mathrm{mV}$; the presences of amine groups in the APTES and CS are the cause of this behavior. The system reported by Wang et al., in which sodium ascorbate was used as a reducing agent, presents a $\zeta$ potential $+30 \mathrm{mV}$ [42]. The presence of a polymeric layer of CS on the surface of the QDs causes this difference. A positive character promotes cell internalization due to the electrostatic interaction between the QDs (with a lively character) and the lipid bilayer of the cell wall (with a negative character) [44]. This positive character is a desirable characteristic in cellular markers that require access to the cytosol. 


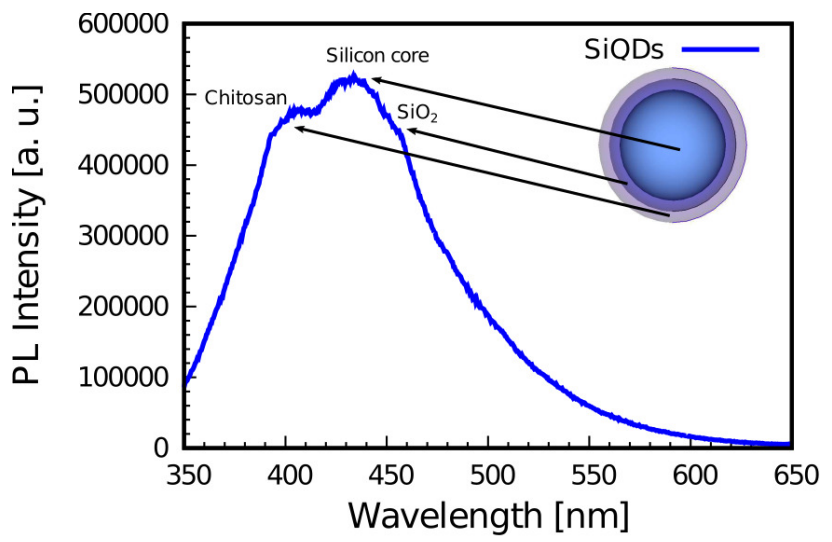

FIGURE 4. Photoluminescence spectrum of the SiQDs.

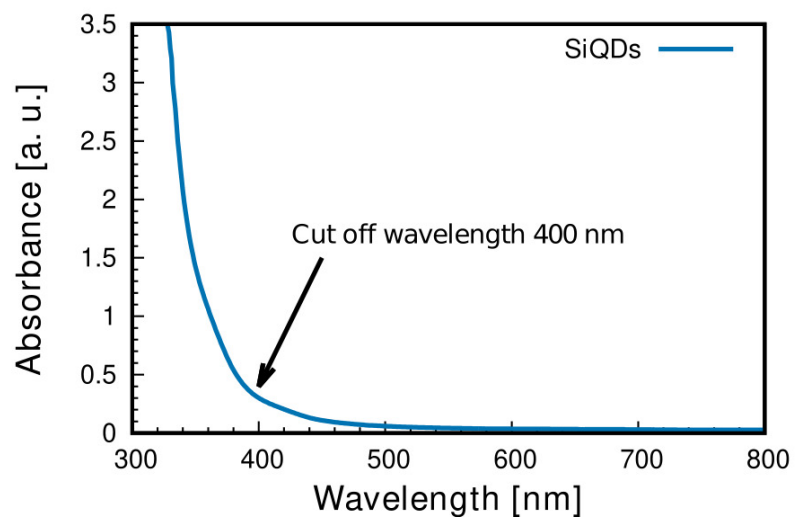

FIGURE 5. UV-Vis absorption spectrum of the SiQDs.

\subsection{Photoluminescence}

Figure 4 shows the photoluminescence spectrum of the SiQDs at room temperature. We can observe the two characteristic emissions of the silicon core and the $\mathrm{SiO}_{2}$ at 434.5 $\mathrm{nm}(2.85 \mathrm{eV})$ [45] and $447.5 \mathrm{~nm}(2.77 \mathrm{eV})$ [46], respectively. The CS causes the third emission in $407.5 \mathrm{~nm}(3.04 \mathrm{eV})$, according to several works $[47,48]$. Therefore, we can propose a polymer layer of CS on the surface of the QD. The presence of CS increases the nanoparticle's biocompatibility [49,50], which is highly desirable in biomedical applications. The figure inset shows a proposed schematic diagram of the kind, composition, and shape of the SiQDs.

\subsection{Absorbance and band gap}

Figure 5 shows the absorption spectrum of the SiQDs. The synthesized SiQDs show an absorption spectrum showed a robust cut-off wavelength at $400 \mathrm{~nm}$. PerkinElmer's simple method [51] and the band gap estimation was by the following Eq. (1) using the experimental absorbance values.

$$
\operatorname{Energy}(E)=h * C / \lambda
$$

where: $h$ is the Planck's constant $h=6.626 \times 10^{-34} \mathrm{~J} \cdot \mathrm{s}$, $C=$ Speed of light $=3.0 \times 10^{8} \mathrm{~m} / \mathrm{s}, \lambda$ cutoff wavelength

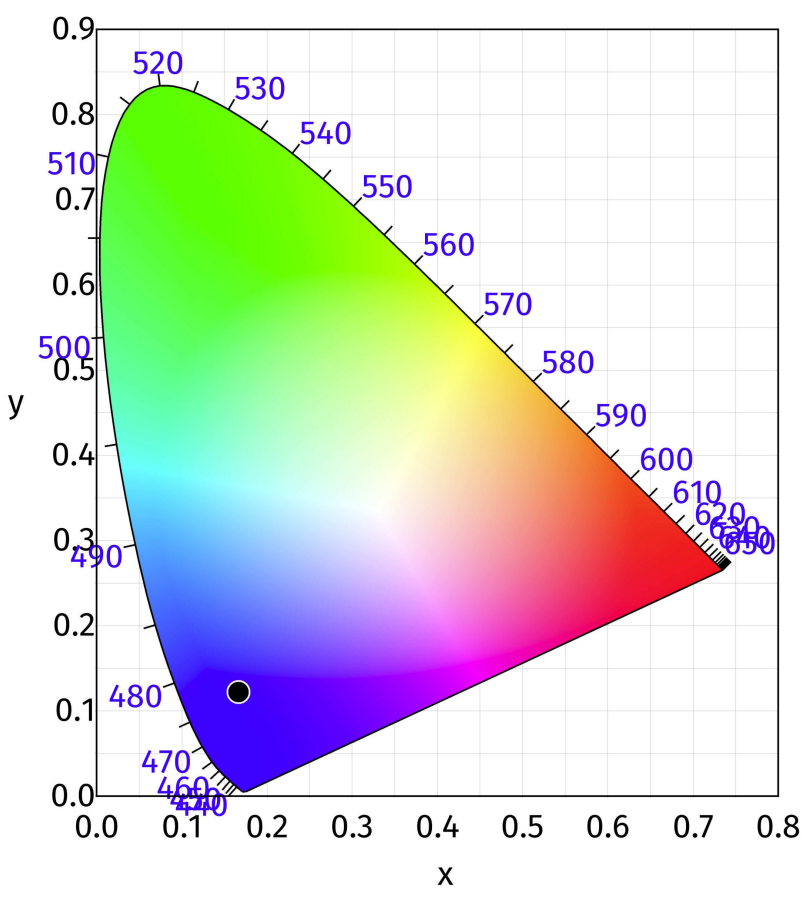

FIGURE 6. CIE 1931 chromaticity coordinates of SiQDs.

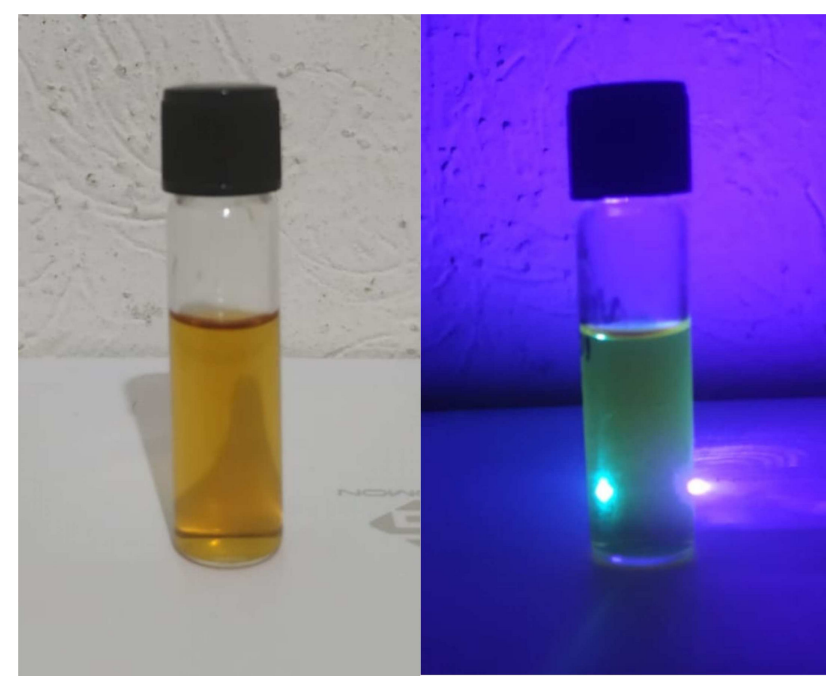

FIGURE 7. SiQDs under UV light.

$\lambda=400 \times 10^{-9} \mathrm{~m}$ and $1 \mathrm{eV}=1.6 \times 10^{-19} \mathrm{~J}$ (conversion factor). The estimated value obtained for the band gap was $3.1 \mathrm{eV}$.

\subsection{Comission Internationale de l'Éclairage 1931 chro- maticity coordinates}

The PL response of the SiQDs was characterized by CIE 1931 chromaticity coordinates [52], as shown in Fig. 6, obtained using the photoluminescence spectrum and the software ColorCalculator by OSRAM Sylvania, Inc. [53]. The SiQDs emit blue light $(x=0.1665, y=0.1222)$ under UV radiation as shown in Fig. 7. This emission offers potential 


\begin{tabular}{ccccc}
\hline \hline TABLE I. Comparison of SiQDs properties for similar synthesis pathways. & & \\
\hline Research's authors & $\begin{array}{c}\text { Size distribution } \\
\text { (DLS) }\end{array}$ & Photoluminescence & Silicon source & Reductor agent \\
\hline P.A. Hernandez-Abril, & $\sim 2.3 \mathrm{~nm}$ & $447.5 \mathrm{~nm} 434.5 \mathrm{~nm}$ & APTES & Low molecular weight chitosan \\
et al. & $\sim 2.8 \mathrm{~nm}$. & and $407.5 \mathrm{~nm}$. & Sodium Ascorbate \\
Jing Wang, et al. [8]. & $\sim 3-5 \mathrm{~nm}$. & $530 \mathrm{~nm}$. & APTES & Trisodium citrate \\
Jintai Lin, et al. [56]. & $\sim 3.86 \mathrm{~nm}$. & $460 \mathrm{~nm}$. & APTES & Trisodium citrate dihydrate \\
Yiling Zhong, et al. [57]. & &
\end{tabular}

electronic applications such as down-shifting material on a solar cell's window side to improve the photocurrent generation [54].

The use of CS as a novel silicon reducer for SiQDs synthesis has some advantages when compared to others as shown in Table I. Previous research reports the change particles surface charges and the biocompatibility increase by using CS.

CS has been used to change particles' surface charge and increase their biocompatibility [55]. One of the benefits of using chitosan as a reducer is smaller particle sizes. In this work, CS has a double function: to be the reducing agent and functionalize the surface of the QDs.

\section{Conclusions}

The low-cost synthesis of SiQDs (does not require high priced equipment) and eco-friendly technique (room tem- perature and atmospheric pressure) was successful. In the synthesis, low molecular weight CS was used as a reducing agent. Its photoluminescent properties and size can be used as fluorescent probes, solar cells down-shifting coatings, and bio-sensors. The nanoplatform has a core-shell structure in which the CS is on the surface. The positive $\zeta$ potential increases surface functionalization possibilities for several biomedical applications that require cellular uptakes, such as controlled drug release and cell marker.

\section{Acknowledgments}

The authors express our gratitude to the nanoFAB Laboratory for its facilities, to CeMIE-SOL for the use of characterization equipment, and CONACyT for the basic science project No. 242508 and FORDECyT project No. 272894.
1. Z. Kang et al., Water-Soluble Silicon Quantum Dots with Wavelength-Tunable Photoluminiscence, Adv. Mater. 21 (2009) 661, https://doi.org/10.1002/adma. 200801642 .

2. S. Bhattacharjee, Cytotoxicity of surface-funtionalized silicon and germanium nanoparticles: the dominant role of surface charges, Nanoscale 5 (2013) 4870, https://doi.org/ 10.1039/C3NR34266B

3. S. Ma et al., One-Step Synthesis of Water-Dispersible and Biocompatible Silicon Nanoparticles for Selective Heparin Sensing and Cell Imaging, Anal. Chem. 88 (2016) 10474, https: //doi.org/10.1021/acs.analchem.6b02448.

4. L. T. Canham, Silicon quantum wire array fabrication by electrochemical and chemical dissolution of wafers, Appl. Phys. Lett. 57 (1990) 1046, https://doi.org/10.1063/1. 103561

5. H. J. Higuera-Valenzuela et al., Efficiency enhancement of silicon solar cells by silicon quantum dots embedded in $\mathrm{ZnO}$ films as down-shifting coating, J. Mater Sci.: Mater. Electron. 31 (2020) 20561, https://doi.org/10.1007/ s10854-020-04576-0

6. S. Morozova, M. Alikina, A. Vinogradov, and M. Pagliaro, Silicon Quantum Dots: Synthesis, Encapsulation, and Appli- cation in Light-Emitting Diodes, Front. Chem. 8 (2020) 191, https://doi.org/10.3389/fchem.2020.00191

7. Z. Bisadi et al., Silicon nanocrystals for nonlinear optics and secure communications, Phys. Status Solidi A 212 (2015) 2659, https://doi.org/10.1002/pssa.201532528

8. J. Wang et al., One-step synthesis of water-dispersible silicon nanoparticles and their use in fluorescence lifetime imaging of living cells, J. Mater. Chem. B 2 (2014) 4338, https: //doi.org/10.1039/C4TB00366G

9. Y. Zhong et al., Large-Scale Aqueous Synthesis of Fluorescent and Biocompatible Silicon Nanoparticles and Their Use as Highly Photostable Biological Probes, J. Am. Chem. Soc. 135 (2013) 8350, https://doi.org/10.1021/ ja4026227

10. E. Roduner, Size matters: why nanomaterials are different, Chem. Soc. Rev. 35 (2006) 583, https://doi.org/10. 1039/B502142C

11. S. Furukawa and T. Miyasato, Quantum size effects on the optical band gap of microcrystalline Si:H, Phys. Rev. B 38 (1988) 5726, https://doi.org/10.1103/PhysRevB. 38.5726 
12. K. Hata et al., Self-Assembled Monolayer as a Template to Deposit Silicon Nanoparticles Fabricated by Laser Ablation, $J$. Phys. Chem. B 105 (2001) 10842, https: / / doi.org/10. 1021/jp010760b

13. D. Kovalev et al., Breakdown of the $k$-Conservation Rule in Si Nanocrystals, Phys. Rev. Lett. 81 (1998) 2803, https: //doi.org/10.1103/PhysRevLett.81.2803

14. O. Schoenfeld et al., Formation of Si quantum dots in nanocrystalline silicon, Solid State Electron. 40 (1996) 605, https: //doi.org/10.1016/0038-1101(95)00371-1

15. R. D. Tilley et al., Micro-emulsion synthesis of monodisperse surface stabilized silicon nanocrystals, Chem. Commun. (2005) 1833, https://doi.org/10.1039/B416069J

16. J. P. Wilcoxon, G. A. Samara, and P. N. Provencio, Optical and electronic properties of $\mathrm{Si}$ nanoclusters synthesized in inverse micelles, Phys. Rev. B 60 (1999) 2704, https : / / doi .org/ 10.1103/PhysRevB.60.2704

17. R. A. Bley and S. M. Kauzlarich, A Low-Temperature Solution Phase Route for the Synthesis of Silicon Nanoclusters, J. Am. Chem. Soc. 118 (1996) 12461, https://doi.org/ $10.1021 / j a 962787 \mathrm{~s}$

18. B. Ghosh and N. Shirahata, Colloidal silicon quantum dots: synthesis and luminescence tuning from the near-UV to the near-IR range, Sci. Technol. Adv. Mater. 15 (2014) 014207, https://doi.org/10.1088/1468-6996/15/1/ 014207 .

19. P. Raveendran, J. Fu, and S. L. Wallen, Completely "Green" Synthesis and Stabilization of Metal Nanoparticles, J. Am. Chem. Soc. 125 (2003) 13940, https://doi.org/10. $1021 / j a 029267 j$

20. C. Wang et al., Preparation, Characterization and Application of Polysaccharide-Based Metallic Nanoparticles: A Review, Polymers 9 (2017) 689, https://doi.org/10.3390/ polym9120689.

21. Y. Park et al., Polysaccharides and phytochemicals: a natural reservoir for the green synthesis of gold and silver nanoparticles, IET Nanobiotechnol. 5 (2001) 69, https: / / doi .org/ $10.1049 /$ iet -nbt.2010.0033

22. X. Li et al., Liquid Metal Droplets Wrapped with Polysaccharide Microgel as Biocompatible Aqueous Ink for Flexible Conductive Deviced, Adv. Funct. Mater. 28 (2018) 1804197, https://doi.org/10.1002/adfm.201804197

23. Z. Wei et al., Novel Biocompatible Polysaccharide-Based SelfHealing Hydrogel, Adv. Funct. Mater. 25 (2015) 1352, https: //doi.org/10.1002/adfm.201401502

24. I. C. M. Dea, Industrial polysaccharides, Pure Appl. Chem. 61 (1989) 1315, https://doi.org/10.1351/ pac198961071315a

25. S. Mizrahy and D. Peer, Polysaccharides as building blocks for nanotherapeutics, Chem. Soc. Rev. 41 (2012) 2623, https: //doi.org/10.1039/C1CS15239D

26. C. Gérente et al., Removal of metal ions from aqueous solution on low cost natural polysaccharides: Sorption mechanism approach, React. Funct. Polym. 46 (2000) 135, https : //doi.org/10.1016/S1381-5148(00)00047-X
27. J. Wang et al., Processable and Luminescent Supramolecular Hydrogels from Complex Coacervation of Polycations with Lanthanide Coordination Polyanions, Macromolecules 52 (2019) 8643, https://doi.org/10.1021/acs. macromol.9b01568.

28. M. Abramson, O. Shoseyov, and Z. Shani, Plant cell wall reconstruction toward improved lignocellulosic production and processability, Plant Sci. 178 (2010) 61, https://doi.org/ 10.1016/j.plantsci.2009.11.003

29. A. Travan et al., Non-cytotoxic Silver NanoparticlePolysaccharide Nanocomposites with Antimicrobial Activity, Biomacromolecules 10 (2009) 1429, https://doi.org/10.1021/bm900039x

30. H. Huang and X. Yang, Synthesis of polysaccharide-stabilized gold and silver nanoparticles: a green method, Carbohydr. Res. 339 (2004) 2627, https://doi.org/10.1016/j. carres.2004.08.005.

31. H. Honarkar and M. Barikani, Applications of biopolymers I: chitosan, Monatsh. Chem. 140 (2009) 1403, https : //doi. org/10.1007/s00706-009-0197-4

32. D. A. Alarcón-Payán, R. D. Koyani, and R. VazquezDuhalt, Chitosan-based biocatalytic nanoparticles for pollutant removal from wastewater, Enzyme Microb. Technol. 100 (2017) 71, https://doi.org/10.1016/j. enzmictec.2017.02.008

33. C. Bergonzi et al., Study of 3D-printed chitosan scaffold features after different post-printing gelation processes, Sci. Rep. 9 (2019) 362, https://doi.org/10.1038/ s41598-018-36613-8

34. Z. Amoozgar, J. Park, Q. Lin, and Y. Yeo, Low MolecularWeight Chitosan as a $\mathrm{pH}-$ Sensitive Stealth Coating for TumorSpecific Drug Delivery, Mol. Pharm. 9 (2012) 1262, https: //doi.org/10.1021/mp2005615

35. L. Sun et al., One pot synthesis of gold nanoparticles using chitosan with varying degree of deacetylation and molecular weight, Carbohydr. Polym. 178 (2017) 105, https: //doi. org/10.1016/j.carbpol.2017.09.032

36. E. Susilowati, Maryani, and Ashadi, Sunlight-assisted synthesis of colloidal silver nanoparticles using chitosan as reducing agent, IOP Conf. Ser.: Mater. Sci. Eng. 349 (2018) 012019, https://doi.org/10.1088/1757-899X/349/1/ 012019

37. J. Wu, J. Dai, Y. Shao, and Y. Sun, One-step synthesis of fluorescent silicon quantum dots (Si-QDs) and their application for cell imaging, $R S C A d v .5$ (2015) 83581, https: //doi.org/10.1039/C5RA13119G

38. S. Chinnathambi, S. Chen, S. Ganesan, and N. Hanagata, Silicon Quantum Dots for Biological Applications, $A d v$. Healthc. Mater. 3 (2013) 10, https://doi.org/10. 1002 /adhm.201300157

39. L. W. Zhang and N. A. Monteiro-Riviere, Mechanisms of Quantum Dot Nanoparticle Cellular Uptake, Toxicol. Sci. 110 (2009) 138, https : / doi.org/10.1093/toxsci/ kfp087

40. S. Ohta, S. Inasawa, and Y. Yamaguchi, Real time observation and kinetic modeling of the cellular uptake and removal of silicon quantum dots, Biomateri- 
als 33 (2012) 4639, https://doi.org/10.1016/j. biomaterials.2012.03.029

41. E. Fröhlich, The role of surface charge in cellular uptake and cytotoxicity of medical nanoparticles, Int. J. Nanomed. 7 (2012) 5577, https://doi.org/10.2147/IJN.S36111.

42. Y. Zhong et al., Facile, Large-Quantity Synthesis of Stable, Tunable-Color Silicon Nanoparticles and Their Application for Long-Term Cellular Imaging, ACS Nano 9 (2015) 5958, https://doi.org/10.1021/acsnano.5b00683

43. L.-S. Liao et al., Blue luminescence from $\mathrm{Si}^{+}$-implanted $\mathrm{SiO}_{2}$ films thermally grown on crystalline silicon, Appl. Phys. Lett. 68 (1996) 850, https://doi.org/10.1063/1. 116554

44. X. Pan et al., Photoluminescence from Chitosan for BioImaging, Austr. J. Chem. 67 (2014) 1422, https://doi. org/10.1071/CH14274

45. H. Huang et al., Enhanced fluorescence of chitosan based on size change of micelles and application to directly selective detecting $\mathrm{Fe}^{3+}$ in humanserum, Biosens. Bioelectron. 42 (2013) 539, https://doi.org/10.1016/j.bios. 2012.10 .098

46. R. Duan et al., Chitosan-coated Gold Nanorods for Cancer Therapy Combining Chemical and Phototermal Effects, Macromol. Biosci. 14 (2014) 1160, https://doi.org/ $10.1002 / \mathrm{mabi} .201300563$
47. S.-F. Shi et al., Biocompatibility of chitosan-coated iron oxide nanoparticles with osteoblast cells, Int. J. Nanomed. 7 (2012) 5593, https://doi.org/10.2147/IJN.S34348

48. J. Dharma and A. Pisal, Simple Method of Measuring the Band Gap Energy Value of $\mathrm{TiO}_{2}$ in the Powder Form using a UV/Vis/NIR Spectrometer, https://www.perkinelmer.com. Cn/CMSResources/Images/46-74327APP. UVVISNIRMeasureBandGapEnergyValue.pdf

49. A. N. Meza-Rocha, I. Camarillo, R. Lozada-Morales, and U. Caldiño, Reddish-orange and neutral/warm white light emitting phosphots: $\mathrm{Eu}^{3+}, \mathrm{Dy}^{3+}$ and $\mathrm{Dy}^{3+} / \mathrm{Eu}^{3+}$ in potassiumzinc phosphate glasses, J. Lumin. 183 (2017) 341, https: //doi.org/10.1016/j.jlumin.2016.11.068

50. J. Selveria, ColorCalculator, Osram Sylvania, 2020.

51. R. Lopez-Delgado et al., Solar cell efficiency improvement employing down-shifting silicon quantum dots, Microsyst. Technol. 24 (2018) 495, https://doi.org/10.1007/ s00542-017-3405-x

52. R. Duan et al., Chitosan-coated Gold Nanorods for Cancer Therapy Combining Chemical and Photothermal Effects, Macromol. Biosci. 14 (2014) 1160, https://doi.org/ $10.1002 / \mathrm{mabi} .201300563$

53. J. Lin and Q. Wang, Role of novel silicon nanoparticles in luminescence detection of a family of antibiotics, RSC Adv. 5 (2015) 27458, https://doi.org/10.1039/C5RA01769F 